\title{
Evaluation of strong shear thinning non-Newtonian fluid flow using single domain DR-BEM
}

\author{
M. Giraldo ${ }^{1}$, H. Power ${ }^{2}$ \& W. Flórez ${ }^{1}$ \\ ${ }^{1}$ Instituto de Energía y Termodinámica, Universidad Pontificia \\ Bolivariana, Medellín, Colombia \\ ${ }^{2}$ School of Mechanical, Materials and Manufacturing Engineering, \\ University of Nottingham, Nottingham, UK
}

\begin{abstract}
The dual reciprocity method (DRM) has been successfully employed along with the boundary element method (BEM) to simulate non linear flow phenomena such as convective momentum transport and shear thinning fluids. In the latter however, domain partitioning has been necessary to achieve convergence when the power law index is below 0.8. This paper shows how a single domain DR-BEM formulation for non Newtonian low Reynolds number flows can be implemented in order to obtain accurate results for lower values of the power law index. Some of the characteristics of this implementation are the use of quadratic elements and an iterative solution of the non linear system of equations using a modified Newton-Raphson method. Along with the implementation, two radial basis functions (RBFs) were used and compared on two classical problems of inelastic non Newtonian flow: couette mixing and slit flow. Solutions obtained are also compared to results from a multi-domain dual reciprocity method (MD-DRM) for equal meshes. Results showed that using the above mentioned strategies, single domain DR-BEM can accurately predict the flow field in inelastic non Newtonian flow for values of the power law index as low as 0.5 . It is also worth noting that the accuracy of the single domain strategy was shown to be higher than MD-DRM, although the latter clearly reduced computational resource consumption.

Keywords: single domain DRM, power law fluids, higher order elements, iterative methods for non linear systems.
\end{abstract}




\section{Introduction}

Study of non linear phenomena such as non Newtonian fluid flow using domain methods is accurate but requires large amounts of computational resources and are often accompanied with complex remeshing algorithms. The BEM has the advantage of greatly reducing the computational cost of numerical simulations by only discretizing the boundary of the problem thus reducing the problem dimensionality by one [1], but are often seen in disadvantage to domain methods when dealing with non linear problems. Non linear terms are usually treated in BEM as a domain integral of a pseudo body force [2], that was originally solved using cell integration, a type of domain meshing that makes BEM lose its boundary only nature and increase computational cost beyond FEM [3]. A more recent development is the DRM which uses RBF interpolation to expand the non linear term and then apply the usual divergence theorem to convert domain integrals into equivalent boundary integrals [4].

The main difficulty associated with shear thinning and shear thickening fluids is the dependence of the viscosity on domain variables, namely velocity gradients, leading to highly non linear Partial Differential Equation (PDF). To solve these type of flows, [2] uses a DRM approximation along with domain sub divisions. The resulting approach called multi-domain dual reciprocity (MD-DRM) showed to have good accuracy while significantly reducing resource consumption in comparison to other subdomain approaches such as the Green element method (GEM) [5]. Both works coincide with in the necessity of subdomains for the simulation of highly non linear problems.

The objective of this paper is to show how an adequate implementation of the DR-BEM along with a specific iterative scheme for the non linear system of equations can give accurate results for the flow of an inelastic non Newtonian fluid obeying the Power Law rheological model [6] without the need of domain partitioning, situation that can be an advantage if moving boundaries are considered. Two numerical examples are used to test the performance of the proposed formulation: couette and slit flow, both having analytical solutions to which the results obtained are compared [6]. The use of these examples also allows for different considerations as the first has only Dirichlet boundary conditions while the second is a mixed boundary condition problem (pressure driven flow). This implementation is also tested using two different radial basis functions (RBF), the traditional thin plate spline (TPS) and a more up-to-date compact support RBF with different values of the compactness parameter.

Work is divided as follows. Governing equations and integral formulation are initially presented, followed by the details of the numerical implementation of the resulting equations. The next section shows the results obtained for the different problems and finally conclusions are drawn. 


\section{Direct boundary integral formulation for non Newtonian fluids}

The fluid inside the a closed Lyapunov surfaces $S$ must obey the non Newtonian Stokes system of equations (1). The use of Stokes equation for this case is supported in the fact that common non Newtonian fluids have viscosities well over $10^{3} \mathrm{~Pa} \cdot \mathrm{s}$, assuring that $R e<<1$.

$$
\frac{\partial u_{i}}{\partial x_{i}}=0 \quad \frac{\partial \sigma_{i j}}{\partial x_{j}}=0
$$

where

$$
\sigma_{i j}=-p \delta_{i j}+\eta(\dot{\gamma})\left(\frac{\partial u_{i}}{\partial x_{j}}+\frac{\partial u_{j}}{\partial x_{i}}\right)
$$

In equations (1) and (2), $\vec{u}$ is the velocity, $p$ the pressure, $\delta_{i j}$ the Kronecker delta, $\eta(\dot{\gamma})$ is the viscosity of the fluid.

An adequate rheological model for $\eta(\dot{\gamma})$ must be selected in order to accurately simulate a given physical system. In the case of inelastic non Newtonian fluids, the power law model has shown to be a powerful tool bringing together accuracy and simplicity [6].

$$
\eta(\dot{\gamma})=k \dot{\gamma}^{n-1}
$$

Boundary conditions vary according to the problem that is being solved. In the case of couette flow, the external cylinder is stationary while the internal one rotates at a constant angular velocity of value 1. For slit flow, the superior and inferior surfaces are stationary, while at the entrance and exit the perpendicular velocities are made nil while the tractions are given only by a pressure difference between them. For the cases shown, $\Delta p$ is also 1 .

Integral representations for the velocity in BEM are only valid for a constant viscosity, therefore a small modification must be made to the internal tractions (2). The idea is to subtract and add the stress tensor multiplied by an arbitrary constant $\mu^{N}$ allowing to redefine the momentum equation as:

$$
\frac{\partial \sigma_{i j}^{N}}{\partial x_{j}}=-\frac{\partial \tau_{i j}^{e}}{\partial x_{j}}
$$

where

$$
\tau_{i j}^{e}=\left(k \dot{\gamma}^{n-1}-\mu^{N}\right)\left(\frac{\partial u_{i}}{\partial x_{j}}+\frac{\partial u_{j}}{\partial x_{i}}\right) ; \sigma_{i j}^{N}=-p \delta_{i j}+\mu^{N}\left(\frac{\partial u_{i}}{\partial x_{j}}+\frac{\partial u_{j}}{\partial x_{i}}\right)
$$

Using Green's second identity for stokes flow [7] to expand the Newtonian traction $\left(\sigma_{i j}^{N}\right)$ and using the properties of the fundamental solutions, the integral representation of the velocity field for an arbitrary point in a closed domain filled 
with a non Newtonian fluid, which for the present case is a point $x \in \Omega_{i}$, is given by:

$$
\begin{aligned}
C u_{i}(x)-\int_{S} K_{i j}(x, y) u_{j}(y) d S_{y} & =\frac{1}{\mu^{N}} \int_{\Omega} u_{i}^{j}(x, y)\left(-\frac{\partial \tau_{j k}^{e}}{\partial x_{k}}\right) d \Omega \\
- & \frac{1}{\mu^{N}} \int_{S} u_{i}^{j}(x, y) \sigma_{j k}^{N}(\vec{u}(y)) n_{k}(y) d S_{y}
\end{aligned}
$$

where $C$ is a constant dependant on the position of the point. For internal points $C=1$ and for point a smooth boundary $C=1 / 2$.

The Stokeslet for two dimensions is given by:

$$
u_{i}^{j}(x, y)=-\frac{1}{4 \pi}\left[\ln \left(\frac{1}{r}\right) \delta_{i j}+\frac{\left(x_{i}-y_{i}\right)\left(x_{j}-y_{j}\right)}{r^{2}}\right]
$$

$r$ being the Euclidean distance between point $x$ and $y, r=|x-y|$. The corresponding normal derivative or Stresslet is given by (in two dimension):

$$
K_{i j}(x, y)=-\frac{1}{\pi} \frac{\left(x_{i}-y_{i}\right)\left(x_{j}-y_{j}\right)\left(x_{k}-y_{k}\right) n_{k}(y)}{r^{4}}
$$

\section{Dual reciprocity approximation}

In order to avoid domain meshing, the dual reciprocity method [4] is used to expand the domain integral. The basis of this method is to approximate the non homogeneous term using interpolation functions [2]:

$$
-\frac{\partial \tau_{j k}^{e}}{\partial x_{k}}=\sum_{m=1}^{p} f\left(x, y^{m}\right) \alpha_{l}^{m} \delta_{i l}
$$

$f\left(x, y^{m}\right)$ is a known set of functions dependent only on geometry and $\alpha_{l}^{m}$ is an unknown vector of coefficients to be determined by collocation on $y^{m}$ ( $m=1,2,3, \ldots, p$ ) points in the domain of interest. Using this approximation and Green's identities, the domain integral becomes:

$$
\begin{aligned}
\int_{\Omega} u_{i}^{j}(x, y) & \left(-\frac{\partial \tau_{j k}^{e}}{\partial x_{k}}\right) d \Omega_{i}=\sum_{m=1}^{p} \alpha_{l}^{m}\left[\hat{u}_{k}^{l}\left(x, z^{m}\right)-\right. \\
& \left.\int_{S} K_{k j}(x, y) \hat{u}_{j}^{l}\left(y, z^{m}\right) d S_{y}+\int_{S} u_{i}^{k}(x, y) \hat{t}_{j}^{l}\left(y, z^{m}\right) d S_{y}\right]
\end{aligned}
$$




\subsection{Choice of interpolating function}

One of the most popular interpolating functions used is the thin plate spline:

$$
f^{m}(x)=r^{2}\left(x, y^{m}\right) \log \left(r\left(x, y^{m}\right)\right)+P_{1}(x)
$$

This function has shown to be an accurate and simple alternative for use with boundary integral methods $[2,3]$. For this case, the augmented polynomial $P_{1}$ is composed of the functions $x_{1}, x_{2}$ and 1 . The particular solutions of the first term of the second degree GTPS are $\left(\bar{x}_{i}=x_{i}-y_{i}^{m}\right)$ :

$$
\begin{gathered}
\hat{u}_{i}^{j}\left(x, y^{m}\right)=\frac{1}{96}\left[\left(5 r^{4} \log r-\frac{7}{3} r^{4}\right) \delta_{i j}-\bar{x}_{i} \bar{x}_{j}\left(4 r^{2} \log r-\frac{5}{3} r^{2}\right)\right] \\
\hat{t}_{i}^{j}\left(x, y^{m}\right)=\frac{1}{96}\left[\begin{array}{l}
8 r^{2}\left(\bar{x}_{j} n_{i}+\bar{x}_{i} n_{j}+\bar{x}_{l} n_{l} \delta_{i j}\right)\left(2 \log r-\frac{1}{3}\right) \\
-4 \bar{x}_{i} \bar{x}_{j} \bar{x}_{l} n_{l}\left(4 \log r+\frac{1}{3}\right)
\end{array}\right]
\end{gathered}
$$

Since the polynomials are an integral part of the GTPS, the particular solutions of the complete GTPS is equal to functions given in (12) and (13) plus the summation of the particular solutions to each of the augmentation polynomials. It is worth noting that these polynomials are only dependant on point $x$, not the other collocation nodes $y^{m}$; this fact also holds true for the auxiliary flow fields.

The values of the auxiliary fields and derivatives of the augmented polynomials used with the second degree GTPS are the following:

For $f(x)=x_{1}$

$$
\begin{array}{r}
\hat{u}_{i}^{j}\left(x, y^{m}\right)=\frac{1}{24}\left[\begin{array}{l}
x_{1}^{3}\left(3 \delta_{i j}-2 \delta_{1 i} \delta_{1 j}-\delta_{2 i} \delta_{2 j}\right)+ \\
3 x_{1} x_{2}^{2}\left(\delta_{i j}-\delta_{1 i} \delta_{1 j}\right)-3 x_{1}^{2} x_{2}\left(\delta_{1 i} \delta_{2 j}+\delta_{2 i} \delta_{1 j}\right)
\end{array}\right] \\
\hat{t}_{i}^{j}(x)=\frac{1}{8}\left[\begin{array}{l}
3 x_{1}^{2}\left(n_{1} \delta_{i j}+n_{j} \delta_{1 i}+n_{i} \delta_{1 j}\right)- \\
2 x_{1}^{2}\left(2 n_{1} \delta_{1 i} \delta_{1 j}+n_{1} \delta_{2 i} \delta_{2 j}+n_{2} \delta_{1 i} \delta_{2 j}+n_{2} \delta_{1 j} \delta_{2 i}\right)+ \\
x_{2}^{2}\left(n_{1} \delta_{i j}+n_{j} \delta_{1 i}+n_{i} \delta_{1 j}-2 n_{1} \delta_{1 i} \delta_{1 j}\right)+ \\
2 x_{1} x_{2}\left(n_{2} \delta_{i j}+n_{j} \delta_{2 i}+n_{i} \delta_{2 j}\right)- \\
4 x_{1} x_{2}\left(n_{2} \delta_{1 i} \delta_{1 j}+n_{1} \delta_{1 i} \delta_{2 j}+n_{1} \delta_{1 j} \delta_{2 i}\right)
\end{array}\right]
\end{array}
$$

Particular solutions for $f(x)=x_{2}$ are of the same form, but wherever a subindex 1 appears, it must be replaced by 2 and vice versa. Finally for $f=1$

$$
\begin{gathered}
\hat{u}_{i}^{j}(x)=\frac{1}{16}\left[3|x|^{2} \delta_{i j}-2\left|x_{i}\right|\left|x_{j}\right|\right] \\
\hat{t}_{i}^{j}(x)=\frac{1}{4}\left[x_{j} n_{i}+x_{i} n_{j}+x_{l} n_{l} \delta_{i j}\right]
\end{gathered}
$$

The second choice of interpolating function is a compact support radial basis function (CS-RBF) [8] which has the characteristic of assigning a value of 0 to points outside a circle of radius $C$. In [9] CS-RBFs are used along with DRM to 
solve the Navier-Stokes system of equations with good results, although as is the case in this paper, the compactness of the function is not used and parameter $C$ is chosen to be bigger than the total domain. The specific function used in this work was (non-zero part):

$$
f^{m}\left(x, y^{m}\right)=\left(1-\frac{r\left(x, y^{m}\right)}{C}\right)^{4}\left(4 \frac{r\left(x, y^{m}\right)}{C}+1\right)
$$

The auxiliary flow fields for this CS-RBF are (Terms corresponding to $r>C$ are omitted):

$$
\begin{gathered}
\hat{u}_{i}^{j}\left(x, y^{m}\right)=\left[\begin{array}{c}
\left(\frac{3}{16} r^{2}-\frac{25}{48} \frac{r^{4}}{C^{2}}+\frac{24}{35} \frac{r^{5}}{C^{3}}-\frac{35}{96} \frac{r^{6}}{C^{4}}+\frac{32}{441} \frac{r^{7}}{C^{5}}\right) \delta_{i j}- \\
\bar{x}_{i} \bar{x}_{j}\left(\frac{1}{8}-\frac{5}{12} \frac{r^{2}}{C^{2}}+\frac{4}{7} \frac{r^{3}}{C^{3}}-\frac{5}{16} \frac{r^{4}}{C^{4}}+\frac{4}{63} \frac{r^{5}}{C^{5}}\right)
\end{array}\right] \\
\hat{t}_{i}^{j}\left(x, y^{m}\right)=\left[\begin{array}{c}
\left(\frac{1}{4}-\frac{5}{3} \frac{r^{2}}{C^{2}}+\frac{20}{7} \frac{r^{3}}{C^{3}}-\frac{15}{8} \frac{r^{4}}{C^{4}}+\frac{4}{9} \frac{r^{5}}{C^{5}}\right) \bar{x}_{j} n_{i}+ \\
\left(\bar{x}_{i} n_{j}+\bar{x}_{l} n_{l} \delta_{i j}\right)\left(\frac{1}{4}-\frac{5}{3} \frac{r^{2}}{C^{2}}+\frac{20}{7} \frac{r^{3}}{C^{3}}-\frac{15}{8} \frac{r^{4}}{C^{4}}+\frac{4}{9} \frac{r^{5}}{C^{5}}\right) \\
-2 \bar{x}_{i} \bar{x}_{j} \bar{x}_{l} n_{l}\left(-\frac{5}{6 C^{2}}+\frac{12}{7} \frac{r}{C^{3}}-\frac{5}{4} \frac{r^{2}}{C^{4}}+\frac{20}{63} \frac{r^{3}}{C^{5}}\right)
\end{array}\right.
\end{gathered}
$$

\section{Integral equation discretization}

Traditionally, the approaches used to approximate both the geometry and the densities have been constant and linear elements. Elements of higher order, such as quadratic, have found only limited application in a reduced number of problems. In this work, both geometrical and functional discretization had been made using quadratic elements. The interpolation scheme for a function $X(\xi)$ is given by:

$$
X(\varepsilon)=\psi_{1}(\varepsilon) X^{(1)}+\psi_{2}(\varepsilon) X^{(2)}+\psi_{3}(\varepsilon) X^{(3)}
$$

where $X^{(1)}, X^{(2)}, X^{(3)}$ are the values of $X(\xi)$ on the three nodes of the element.

Interpolation functions $\vec{\psi}$ are show in (22).

$$
\psi_{1}=\frac{1}{2} \varepsilon(\varepsilon-1) ; \quad \psi_{2}=(1-\varepsilon)(1+\varepsilon) ; \quad \psi_{3}=\frac{1}{2} \varepsilon(\varepsilon+1)
$$

The resulting set of integrals are regular and can be evaluated using standard Gaussian quadratures. In order to avoid the weak singularity present when calculating the integral kernels when integrating over the same element where the source point is located, Telles' transformation is initially used, followed by rigid body motion in the case of the double layer kernel [1].

\section{Iterative solution of the non linear system of equations}

After the integral equations are assembled, a system of equations arises for the variables $u_{i}$ on points $x \in \Omega_{i}$, and either $u_{i}$ or $\sigma_{i j} n_{j}$ on $\xi \in S$ depending on 
boundary conditions. Given the non linear character of this system caused by the dependence of $\tau_{i j}^{e}$ on the nodal velocities, an iterative procedure must be set in place to determine the actual solution. For simplicity a new system of equations is defined as:

$$
[A][\vec{X}]=[\vec{C}]+[\vec{B}(\vec{X})]
$$

where matrix $A$ is constituted with elements of matrices $H$ and $G$ depending on boundary conditions. Vector $X$ are the unknowns and $C$ the boundary conditions multiplied by the corresponding elements of $H$ and $G$, and $B$ the non linear term.

The solution of this complete system of equations can be sought in different ways. Simulations showed that traditional schemes such as Piccard iterations only achieved convergence when the power law index is above 0.8 . In order to achieve convergence in values well below 0.8 as those reported in this paper, a Newton-Raphson method with Backtracking was employed directly on (23) without distinction if a given unknown was a velocity or a traction. Following the usual procedure, the following equation is found, where $W(\vec{X})$ is the function to minimize.

$$
W(\vec{X}) \approx W\left(\vec{X}^{(t)}\right)+\frac{1}{\phi} J_{X}\left(\vec{X}^{(t)}\right) \cdot\left(\vec{X}^{(t+1)}-\vec{X}^{(t)}\right)
$$

The Jacobian matrix $\left(J_{X}\right)$ was calculated numerically using centered finite differences with the data available from iteration $t . \phi$ is a smoothing parameter for the iterative method. The program works initially with $\phi=1$, if the problem diverges, then equation (25) is used.

$$
\phi_{\text {optimal }} \approx\left(\frac{\left\|W\left(\vec{X}^{(t+1)}\right)\right\|^{2}}{\left\|W\left(\vec{X}^{(t)}\right)\right\|^{2}}+1\right)^{-1}
$$

\section{Accuracy assessment}

This section presents two problems, couette and slit flow for non Newtonian power law fluids in order to test the performance of the proposed implementation, along with the RBF's and different values of the support parameter $C$ in the case of the CS-RBF (Eq. 18).

\subsection{Couette flow}

This problem has an analytical solution for the tangential velocity. The analytical solution is given by [6]:

$$
u_{t}=\frac{\omega}{\left(\frac{r_{e}}{r_{i}}\right)^{2 / n}-1} \times \frac{r_{e}^{2 / n}-r^{2 / n}}{r^{(2 / n)-1}}
$$


where $u_{t}$ is the tangential velocity at a radius $r, \omega$ is the angular velocity of the internal cylinder of radius $r_{i}$, and $r_{e}$ is the radius of the external cylinder. $n$ is the Power Law Index of the rheological model. A simple mesh consisting of 24 quadratic elements on the outer surface, 24 quadratic elements on the inner surface and 240 internal collocation points will be employed.

Table 1 shows good results of the numerical method when compared to the analytical solutions. It is worth noting that all simulations generated an error below $1 \%$. Best performance by far was obtained with the CS-RBF using a value of $C=15.0,50 \%$ larger than the maximum distance between two points in the domain. At smaller values of $n$ GTPS showed a greater error compared to CSRBF, but total errors are still within acceptable parameters. In all cases, MD-DRM was the least accurate of the tested approximations, but the time used to find such solutions was significantly smaller than the one for the single domain approaches. It is important to note however that the MD-DRM has been tested for values of $n$ as low as 0.2 with good results, but these require denser meshes.

Table 1: Implementation performance on non Newtonian couette problem.

\begin{tabular}{l|cc|cc|cc} 
& \multicolumn{2}{|c|}{$n=0.8$} & \multicolumn{2}{c|}{$n=0.6$} & \multicolumn{2}{c}{$n=0.5$} \\
& L2 error (\%) & Iter. & L2 error (\%) & Iter. & L2 error (\%) & Iter. \\
\hline GTPS & 0.73868 & 18 & 2.0531 & 10 & 3.048 & 16 \\
CS 10.01 & 0.54706 & 6 & 1.5845 & 13 & 2.4178 & 18 \\
CS 15.0 & 0.5464 & 8 & 1.5797 & 18 & 2.4115 & 20 \\
CS 20.0 & 0.54755 & 7 & 1.5797 & 16 & 2.4106 & 17 \\
MD-DRM & 1.9076 & 6 & 4.7367 & 11 & 6.3728 & 14
\end{tabular}

By graphically comparing the results from the single domain approximation CS 20.0 with the MD-DRM approach (Figure 1(a)) it is clear how the single domain solution is quite more accurate in the zones of greater non linearity (left of the plot). Single domain approaches are in this case the best choice in terms of accuracy, although it is important to consider that given the matrix sparsity of MD-DRM solutions found using this approach are significantly faster allowing for the use of denser meshes with which the solution can improve its accuracy.

\subsection{Slit flow}

An analytical solution is available for the velocity profile [6]:

$$
u_{1}=\frac{n}{2 n+2}\left(\frac{1}{2 k} \frac{\Delta p}{L}\right)^{1 / n} h^{(1 / n)+1}\left[1-\left(\frac{2\left|x_{2}\right|}{h}\right)^{(1 / n)+1}\right]
$$




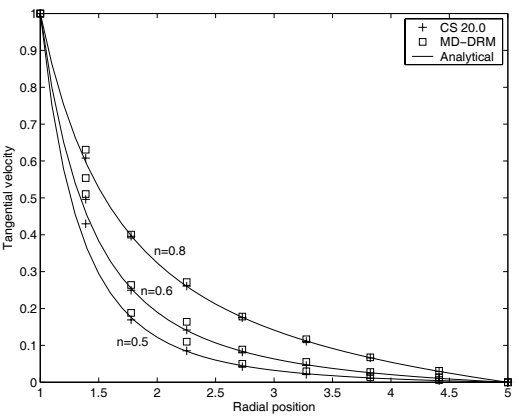

(a)

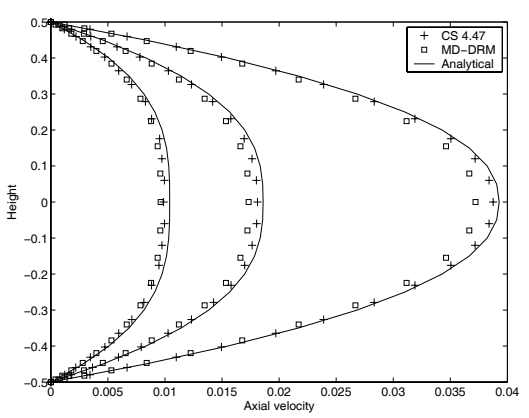

(b)

Figure 1: Velocity profiles for Non Newtonian (a) couette and (b) slit flow.

where $L$ is the channel length, $h$ its height and $\Delta p$ the imposed pressure difference. The tested mesh consisted of 48 quadratic elements on the outer surface, 385 internal collocation points.

Table 2 shows that all the single domain solutions exceed the accuracy of MDDRM for $n=0.8$. The case of CS 3.35 (50\% larger than the maximum distance between two points in the domain) is worth signaling out, as the total L2 error is an order of magnitude below the remaining approximations. As the power law index is reduced, as expected all solutions increase their L2 errors, making some of the single domain approaches show higher errors than MD-DRM for $n=0.5$ but overall showing that the correct selection of the interpolation function can give very accurate results without domain partitioning. However, it is evident that the DR-BEM works better of Dirichlet boundary conditions (i.e. couette flow) than for mixed boundary conditions.

In Figure 1(b) it can be seen that in this case the biggest errors for the single domain approaches are located in the center of the domain, not near the walls where the higher gradients exists. This situation shows that a single domain DRBEM tends to overshot the viscosity increase and therefore decrease the total flow compared to the analytical solutions. As in the former section, the single domain approach has a greater accuracy than MD-DRM.

\section{Conclusions}

The use of single domain DRM for inelastic non Newtonian flows has been traditionally considered not accurate for values of the power law index below 0.8 making the use of domain partitioning a requirement. This paper shows how the use of higher order elements combined with an adequate iteration scheme for the resulting non linear system of equations can improve the results obtained for this problem without the need of domain partitioning. Results for couette and slit flow showed that the use of this strategies improves the accuracy of single domain DRM above that of MD-DRM for values of the power law index as low as 0.5. Even 
Table 2: Implementation performance on Non Newtonian slit flow.

\begin{tabular}{l|cc|cc|cc} 
& \multicolumn{2}{|c|}{$n=0.8$} & \multicolumn{2}{c|}{$n=0.6$} & \multicolumn{2}{c}{$n=0.5$} \\
& L2 error (\%) & Iter. & L2 error (\%) & Iter. & L2 error (\%) & Iter. \\
\hline GTPS & 6.0912 & 6 & 20.457 & 9 & 35.693 & 34 \\
CS 2.24 & 1.3936 & 30 & 10.557 & 13 & 54.355 & 19 \\
CS 3.35 & 0.10092 & 37 & $173.73^{1}$ & 16 & 2.2979 & 21 \\
CS 4.47 & 3.8419 & 20 & 8.9166 & 22 & 16.016 & 16 \\
MD-DRM & 11.238 & 6 & 15.52 & 7 & 18.144 & 8
\end{tabular}

though MD-DRM is less time consuming and can simulate cases of lower values of $n$, the requirement of remeshing if moving boundaries are considered makes the use of single domain DRM a justified and accurate choice.

\section{References}

[1] Brebbia, C., Telles, J. \& Wrobel, L., Boundary Element Techniques. Springer Verlang: New York, 1984.

[2] Flórez, W. \& Power, H., Multi-domain mass conservative dual reciprocity method for the solution of the non-newtonian stokes equations. Applied Mathematical Modelling, 26, pp. 397-419, 2002.

[3] Power, H. \& Botte, V., An indirect boundary element method for solving low reynolds number navier-stokes equations in a three-dimensional cavity. International Journal for Numerical Methods in Engineering, 41, pp. 14851505, 1998.

[4] Partridge, P., Brebbia, C. \& Wrobel, L., The dual reciprocity boundary element method. Computational Mechanics Publications: Southampton, 1992.

[5] Tran-Canh, D. \& Tran-Cong, T., Bem-nn computation of generalised newtonian flows. Engineering Analysis with Boundary Elements, 26, pp. 1528, 2006.

[6] Bird, R.B., Armstrong, R.C. \& Hassager, O., Dynamics of polymeric liquids, volume 1: Fluid mechanics. John Wiley \& sons: New York, 2nd edition, 1987.

[7] Ladyzhensaya, O., The mathematical theory of viscous incompressible flow. Gordon and Breach: New York, 1963.

[8] Chen, C., Brebbia, C. \& Power, H., Dual reciprocity method using compactly supported radia basis functions. Communications in numerical methods in engineering, 15, pp. 137-150, 1999.

[9] Shuaib, N.H., Numerical simulation of thin film flow including shear and gravitational effects. Phd, University of Nottingham, 2005. 